including length of stay, treatment, mortality, and relapses were analysed and compared to standard hospital episode statistics (HES). Results The annualised hospital incidence of CDAD was 70/20000 admissions, age range $2-100$ yrs (mean $75.5, \mathrm{M}: \mathrm{F}$ ). $76.8 \%$ patients were older than $70 \mathrm{yrs}$. $43 \%$ had received antibiotics prior to admission and 35 (62\%) patients were commenced on antibiotics in hospital. The top 5 were Amoxicillin, Co-amoxiclav, Flucloxacillin, Cephalexin and Trimethoprim. $62 \%$ of these had received one course of antibiotic, and $30 \%$ two or more courses. $39 \%$ patients had a previous admission to hospital in the preceding 12 weeks, $46.4 \%$ were taking a PPI and $35.7 \%$ a laxative. $C$ difficile was confirmed by both toxin and GDH positivity in $80.4 \%$, and by toxin positivity only in $19.6 \%$. Total length of stay ranged from 51 days (16\%). A positive diagnosis was made in $80 \%$ patients, but severity was not always recorded. Stool charts were completed in $70 \%$, serum lactate checked in $10 \%$ and abdominal x-ray done in $30 \%$. Only $25 \%$ patients were seen by an MDT member. $78.5 \%$ pts were treated, $30 /$ 44 (68\%) with Metronidazole and 11/44 (25\%) with Vancomycin as first line drugs. $3 / 44$ patients received both drugs initially. $30.4 \%$ pts received $\leq 7$ days, $50 \%$ upto 14 days and $19.6 \% \geq 14$ days treatment. All cause mortality was $25 \%$, almost entirely in the elderly. $7 \%$ had a recurrence, all treated by Vancomycin and pulsed/tapered regimes and probiotics were used infrequently.

Conclusion CDAD continues to be an important hospital acquired infection with a significant increase in hospital length of stay and high mortality rates, especially in the elderly. This study indicates that a significant proportion of $\mathrm{CDAD}$ may be acquired in the community. Adherence to national recommendations for management and involvement of the MDT needs to be encouraged to improve outcomes.

Competing interests None declared.

\section{PWE-099 CONTRIBUTION OF SURGERY AND DISEASE SUBTYPE TO HEALTH RELATED QUALITY OF LIFE IN PATIENTS WITH LOCALLY ADVANCED AND RECURRENT COLORECTAL CANCER}

doi:10.1136/gutjnl-2012-302514d.99

${ }^{1} \mathrm{P}$ G Vaughan-Shaw, ${ }^{*} \mathrm{R}$ Sreekumar, ${ }^{1} \mathrm{~N} \mathrm{~J}$ Curtis, ${ }^{1} \mathrm{M} \mathrm{D}$ Bullock, ${ }^{2} \mathrm{~T}$ Armstrong, ${ }^{3} \mathrm{~A}$ Bateman, ${ }^{4} \mathrm{~T}$ Bryant, ${ }^{1} \mathrm{~J} \mathrm{~S}$ Knight, ${ }^{5} \mathrm{M} \mathrm{C}$ Hayes, ${ }^{5} \mathrm{R}$ W Lockyer, ${ }^{6} \mathrm{M}$ Phillips, ${ }^{1}$ A H Mirnezami. 'Department of Lower GI Surgery, University Hospital Southampton NHS Foundation Trust, Southampton, UK; ${ }^{2}$ Department of Hepatobiliary Surgery, University Hospital Southampton NHS Foundation Trust, Southampton, UK; ${ }^{3}$ Cancer Sciences, University of Southampton, Southampton, UK; ${ }^{4}$ Department of Radiology, University Hospital Southampton NHS Foundation Trust, Southampton, UK; ${ }^{5}$ Department of Urology, University Hospital Southampton NHS Foundation Trust, Southampton, UK; ${ }^{6}$ Department of Vascular Surgery, University Hospital Southampton NHS Foundation Trust, Southampton, UK

Introduction Management of patients with locally advanced (LA) and locally recurrent (LR) colorectal cancer is challenging, with patients frequently requiring complex multimodality interventions. Despite increasing emphasis on evaluation of health related quality of life (HRQoL) in patients having complex interventions, HRQoL information on patients with LA and LR colorectal cancer is sparse. The aim of this study was to prospectively assess outcome measures and HRQoL in a cohort of patients with LA and LR colorectal cancer at our institution.

Methods LA disease was defined as tumour requiring extended multi-visceral resection in the abdomen or pelvis to achieve an R0 resection. 45 consecutive patients were prospectively assessed over a 2-year period. Demographic, treatment, and cancer-related outcomes were recorded on all patients. Pelvic disease was staged using Leeds and Royal Marsden Hospital classification systems. HRQoL was prospectively evaluated using the EORTC generic and disease specific instruments OLQ-CR30 and OLO-CR29.
Results Median age was 69 (range 46-89) and 60\% were male (27/ 45). There were 25 cases of LA disease and 20 LR cases. 35 patients underwent surgery while 10 patients had non-surgical palliation. R0 resection rate was $94 \%$. HRQoL data were available on 41 patients. Median global health status was 65 (95\% CI 55.5 to 74.1), physical functioning 77 (95\% CI 69.9 to 84.5), and social functioning 72 (95\% CI 60.3 to 83.8), which compared favourably with published EORTC reference values. Global health status and pain were significantly better in patients having surgery compared to non-surgical palliation $(\mathrm{p}<0.0001$ and $\mathrm{p}<0.0004)$. Assessment of individual function and symptom scales revealed no significant difference in HRQoL between LA and LR except for greater buttock pain in patients with LR disease. Conclusion In carefully selected patients, HRQoL after radical multimodality treatment for LA and LR colorectal cancer is acceptable, and gives better results than palliation.

Competing interests None declared.

\section{PWE-100 REPEAT TWO WEEK WAIT REFERRALS FOR SUSPECTED COLORECTAL CANCER}

doi:10.1136/gutjnl-2012-302514d.100

P G Vaughan-Shaw, ${ }^{*}$ J Cutting, N R Borley, J M Wheeler. Department of Colorectal Surgery, Gloucestershire Hospitals NHS Foundation Trust, Cheltenham, UK

Introduction The two week-wait pathway for suspected colorectal cancer (CRC2ww) ensures prompt review and investigation of patients with symptoms concerning for malignancy. Previous investigation of such patients does not preclude future repeat referral yet may not represent efficient use of limited clinic time or diagnostic services. This study aims to evaluate the incidence and outcome of repeat CRC2ww referrals.

Methods A retrospective review of all CRC2ww referrals to our unit over a 4-year period was conducted. Patients with previous CRC2ww referrals were identified from a hospital database. Referral indication and outcome for all referrals was collected.

Results 2731 CRC2ww referrals were made between July 2007 and July 2011, median age 72 (19-102), 1179 (43\%) male. 273 cancers were identified including 212 colorectal cancers. 121 referrals were repeat referrals, with 77 made prior to July 2007, and a median 1087.5 (35-2709) days between initial and repeat referrals. Referral indication was the same in $55(47 \%)$ cases. When compared to unique referrals, repeat referral were associated with increased age ( 79 years vs 71 years, $p<0.0001)$ and increased proportion of females $(69 \%$ vs $56 \%$, $\mathrm{p}=0.0048)$. Six cancers, including two CRC, were identified following repeat referral with a median 1511.5 (477-1988) days between initial and repeat referrals. There was no statistical difference in cancer detection between unique or repeat referrals ( $5.2 \%$ vs $10.4 \%, p=0.07$ ). Conclusion Repeat referrals comprise a small proportion of all CRC2ww referrals. Such patients are older and more commonly female. However, cancer detection is not significantly lower in this cohort when compared to those not previously referred. Historical referral or investigation should not preclude future CRC2ww referrals and such patients should be investigated to the same extent as unique referrals.

Competing interests None declared.

\section{PWE-101 HIGH DEFINITION ENDOSCOPY INCREASES THE NUMBER OF ADENOMAS DETECTED IN THE UK BOWEL CANCER SCREENING POPULATION}

doi:10.1136/gutjnl-2012-302514d.101

P J Basford, * S Tholoor, J Homer, P Bhandari. Department of Gastroenterology, Portsmouth Hospitals NHS Trust, Portsmouth, UK

Introduction Adenoma detection and removal is one of the main goals of colonoscopy. Improved adenoma detection has been shown 